\title{
Demethylbelamcandaquinone B Isolated from Labisia pumila Enhanced Proliferation and Differentiation of Osteoblast Cells
}

\author{
Haryati Ahmad Hairi ${ }^{1}$, Jamia Azdina Jamal ${ }^{2}$, Nor Ashila Aladdin², Khairana Husin², Noor Suhaili Mohd Sofi ${ }^{1}$, \\ Norazlina Mohamed $^{1}$, Isa Naina Mohamed ${ }^{1}$, Ahmad Nazrun Shuid ${ }^{1 *}$ \\ ${ }^{1}$ Department of Pharmacology, Faculty of Medicine, Preclinical Building, Universiti Kebangsaan Malaysia, Jalan Yaacob Latiff, Bandar Tun Razak, Cheras, \\ 56000 Kuala Lumpur, Malaysia. \\ ${ }^{2}$ Faculty of Pharmacy, Universiti Kebangsaan Malaysia, Jalan Raja Abdul Aziz, 50300 Kuala Lumpur, Malaysia.
}

\section{ARTICLE INFO \\ Article history: \\ Received on: 19/05/2018 \\ Accepted on: 19/07/2018 \\ Available online: $31 / 08 / 2018$}

\section{Key words:}

active compound, Labisia

pumila, osteoporosis,

osteoblasts.

\begin{tabular}{l}
\hline ABSTRACT \\
\hline Labisia pumila (LP) or more commonly known as Kacip Fatimah in Malaysia has received much attention due to its \\
estrogenic effects, including its role in the treatment of osteoporosis. This study was designed to explore the active \\
compound of LP that may be responsible for its anti-osteoporotic effects. Crude aqueous extract of Labisia pumila \\
var alata (LPva) was fractionated into hexane (Hex), dichloromethane (DCM) and methanol (Met) solvents and \\
their proliferative effects on mouse osteoblastic cell line (MC3T3-E1) were evaluated with MTS bioassay. The DCM \\
fraction significantly promoted cell proliferation in a dose-dependent manner. Thin layer chromatography (TLC) was \\
performed on the DCM fraction of LPva to separate the constituents and the potential active compound was identified. \\
Further isolation was achieved by column chromatography (CC) and Sephadex LH-20 column chromatography. The \\
bioactivity of the isolated compound was confirmed by its ability to replicate MC3T3-E1 accelerated proliferation \\
and differentiation. Mass spectrometry and nuclear magnetic resonance (NMR) identified the active compound as \\
demethylbelamcandaquinone B. Further studies are required to determine the potential of this active compound of \\
LPva in treating osteoporosis.
\end{tabular}

\section{INTRODUCTION}

Osteoporosis, a worldwide prevalence of disease, can be defined as a systemic skeletal disease characterized by weak bones and high risk of fractures (Kanis and Johnell, 2006). This disease generally affects the elderly population and is associated with postmenopausal estrogen deficiency and progressive bone loss (McCann et al., 2008; Pisani et al., 2016). Low estrogen level leads to excessive bone resorption and reduced bone formation (Bord and Beavan, 2003; Sorensen et al., 2006). The imbalance of bone remodeling resulted in a bone loss and eventually osteoporosis (Feng and Mcdonald, 2011). The main option for prevention and treatment of postmenopausal osteoporosis is hormone replacement

\footnotetext{
"Corresponding Author Ahmad Nazrun Shuid, Department of Pharmacology, Faculty of Medicine, Pre clinical Building, Universiti Kebangsaan, Malaysia, Jalan Yaacob Latiff, Bandar Tun Razak, Cheras, 56000 Kuala Lumpur. E-mail: anazrun@yahoo.com
}

therapy (HRT). However, HRT was linked to breast carcinoma (Kotsopoulos et al., 2016), endometrial cancer (Chlebowski et al., 2016) and cardiovascular disease (Bassuk and Manson, 2016). Bisphosphonate is a synthetic drug used to reduce the risk of vertebral and hip fractures, but daily bisphosphonate therapy may cause esophagitis and osteonecrosis of the jaw (Xu et al., 2013). Selective estrogen receptor modulator or SERM is another synthetic drug that binds to the intracellular estrogen receptors in target organs as agonists or antagonists. Unfortunately, it may also cause serious side effects such as thromboembolic disorders and hot flushes (Maximov et al., 2013).

Recently, phytoestrogen became a favorable alternative therapeutic agent for treatment of osteoporosis. Phytoestrogens, such as isoflavones (Deyhima et al., 2003; Singh et al., 2015), lignans (Zhu et al., 2017) and coumestans (Ueda et al., 2011) acted as SERMs to exert therapeutic effects against postmenopausal osteoporosis. These phytoestrogens could also promote calcium absorption or regulate bone remodeling through estrogen receptor 
pathway (Guo et al., 2012; Singh et al., 2015; Zhu et al., 2017). They have fewer side effects and suitable for long-term use compared to synthesized drugs (Atteritano et al., 2009; Singh et al., 2015).

Labisia pumila (Blume) Fern-Vill (synonym: Marantodes pumilum (Blume) Kuntze) from Primulaceae family is locally known in Malaysia as Kacip Fatimah. Other common names are kunci Fatimah, selusoh Fatimah, rumput Fatimah and akar Fatimah. There are three types of Labisia pumila (LP), which are var alata, var pumila and var lanceolata (Stone, 1988). A decoction of the whole plant of LP is often used in Southeast Asia folk medicine to treat menstrual irregularities, improve fertility, facilitate childbirth and as postpartum medicine (Muhammad and Mustafa, 1994). Several bioactive compounds in aqueous extract of LP have been reported including flavonoids, ascorbic acid, beta-carotene, anthocyanin and phenols (Mohamad et al., 2009). Recently, LP extract was found to exhibit a number of biological activities including antioxidant, anti-inflammatory (Sanusi et al., 2013), antimicrobial (Karimi et al., 2011), antifungal (Karimi et al., 2013) and antinociceptive (Samuagam et al., 2011). To date, LP received considerable attention since it was proven to have phytoestrogenic properties. LP has the ability to displace estradiol and bind to antibodies raised against estradiol (Wahab et al., 2011). LP was also shown to increase estrogen and testosterone levels and suppress follicle stimulating hormone (FSH) and luteinizing hormone (LH) in ovariectomised rats (Wahab et al., 2011). These actions emulate endogenous estrogen. The phytoestrogenic effects of LP meant that it could counteract various estrogen-deficiency related diseases such as insulin resistance, cardiovascular diseases, and osteoporosis. The phytoestrogenic properties of LP were proven by its uterotropic effects and body weight regulation by modulating secretions of leptin and resistin, and expression of adipokines in adipose tissue (Fazliana et al., 2009).

However, few studies were conducted aiming at the identification and characterization of the active compound that increases bone formation have not been carried out. Hence, in the present study, we aimed for the separation and isolation of the distinct active compound of Labisia pumila var. alata (LPva) which is relevant for anti-osteoporosis treatment using bio-guided fractionation and purification method. This method is a procedure whereby an extract is fractionated and chromatographically re-fractionated until a pure bioactive is isolated.

\section{MATERIALS AND METHODS}

\section{Materials}

The following instruments were used: ATRFTIR PerkinElmer GX (Waltham, MA, USA), HRESI-MS BrukerMicrOTOF-Q 86 (Billerica, MA, USA) and FT-NMR 600 $\mathrm{MHz}$ CryoprobeBrukerAvance III (Billerica, MA, USA). The following adsorbents were used for fractionation and purification, including Merck Si-gel 60 (40-63 $\mu \mathrm{m}$, cat.no. 1.09385) for column chromatography (CC), Sephadex LH-20 for gel permeation chromatography and Merck Kieselgel 60 F254.025 mm (cat. no. 1.05554) for thin layer chromatographic (TLC) analysis. Solvents used were analytical grade and purchased from Merck (Darmstadt, Germany).

\section{Extraction and isolation of compounds}

The voucher specimen of Labisia pumila var. alata (LPva) is UKMB 30006/SM 2622 which were deposited in the Herbarium of Universiti Kebangsaan Malaysia. The extraction of LPva and isolation of the compound were prepared according to (Nor Ashila et al., 2016; Avula et al., 2011) previously with slight modification. The air-dried leaves of LPva (8720 g) with a ratio of dried leaves:water of 1:30 were extracted at the temperature of $60^{\circ} \mathrm{C}$ for 2 hours followed by freeze-drying to obtain a crude aqueous extract with the net yield of $700 \mathrm{~g}$. Part of the crude aqueous extract $(200 \mathrm{~g})$ was successively partitioned with an equal volume of hexane (Hex), dichloromethane (DCM) and methanol (Met) under reflux for 4 hours. The net yield of Hex fraction was $1.5 \mathrm{~g}$; DCM fraction was $1.3 \mathrm{~g}$; Met fraction was $39.83 \mathrm{~g}$ and the balanced residue was $157.39 \mathrm{~g}$. A portion of DCM fraction (1.3 g) was subjected to Si-gel column chromatography and separated with hexane-ethyl acetate (EtOAc) of increasing percentage of EtOAc to afford 15 sub-fractions (1-15). Sub-fraction 8 (81.9 $\mathrm{mg}$ ) was further separated using Sephadex LH-20 and eluted with $1 \% \mathrm{MeOH}$ in chloroform $\left(\mathrm{CHCl}_{3}\right)$ to yield 10 sub-fractions. Sub-fractions I and J (53.6 mg) were combined based on TLC analysis. The combined fraction was purified using Si-gel column chromatography and eluted isocratically with $\mathrm{CHCl}_{3}$-EtOAc $(90: 10)$ to yield pure interest compound (35 mg) (Figure 1).

\section{Cell culture}

Mouse pre-osteoblast cells (MC3T3-E1 cells) (ATCC, Rockville, MD, USA) were grown in modified alpha minimal essential medium ( $\alpha$-MEM) containing $10 \%$ FBS and $1 \%$ antibiotic-antimycotic (Gibco Life Technologies, Inc., Grand Island, NY, USA). Culture media were changed to differentiation medium, $\alpha$-MEM supplemented with $50 \mu \mathrm{g} / \mathrm{ml}$ ascorbic acid and $10 \mathrm{mM} \beta$-glycerophosphate (Sigma, St. Louis, MO, USA) to induce osteoblast differentiation (Yan et al., 2014).

\section{Cell viability}

For cell viability assay, two-time points, 24 hours and 72 hours, were selected for proliferation phase (rapid increase in cell number until confluence) and the cells were initiated for differentiation when proliferation had arrested. Cell viability study was performed using the MTS assay (Promega, Madison, WI, USA) (Lina Wati et al., 2017). Crude aqueous extract of LPva, hexane, DCM and methanol fractions of LPva extract, demethylbelamcandaquinone $\mathrm{B}$ and 17-beta-estradiol (positive control) were analyzed for their proliferative effects on mouse osteoblastic cell line (MC3T3-E1). Fractions and active compounds were dissolved in $1.0 \mathrm{ml}$ dimethylsulfoxide (DMSO) (Sigma, St. Louis, MO, USA). The final culture concentration of DMSO ranged from $0.1 \%$ to $0.001 \%$. $17-\beta$ estradiol (Sigma, St. Louis, MO, USA) was dissolved in ethanol with the final concentration of ethanol around $0.05 \%$. During the experiment, stock solutions were diluted at various concentrations with the differentiation media. Cells were plated at $1 \times 10^{4}$ in 96 -well plate and incubated overnight. Following that, the medium was replaced with differentiation media containing the various concentrations of LPva extract and incubated for 24 hours at $37^{\circ} \mathrm{C}$, in $5 \% \mathrm{CO}_{2}$. After incubation, $20 \mu \mathrm{L}$ MTS was added and cells were further incubated for 2 hours. The absorbance of MTS formazan formed 
was measured at $490 \mathrm{~nm}$ with a microtiter plate reader (Tecan, Austria). The viability assay was performed to obtain the optimum dose of LPva treatment for the subsequent experiment. This viability assay was repeated again with its active compound.

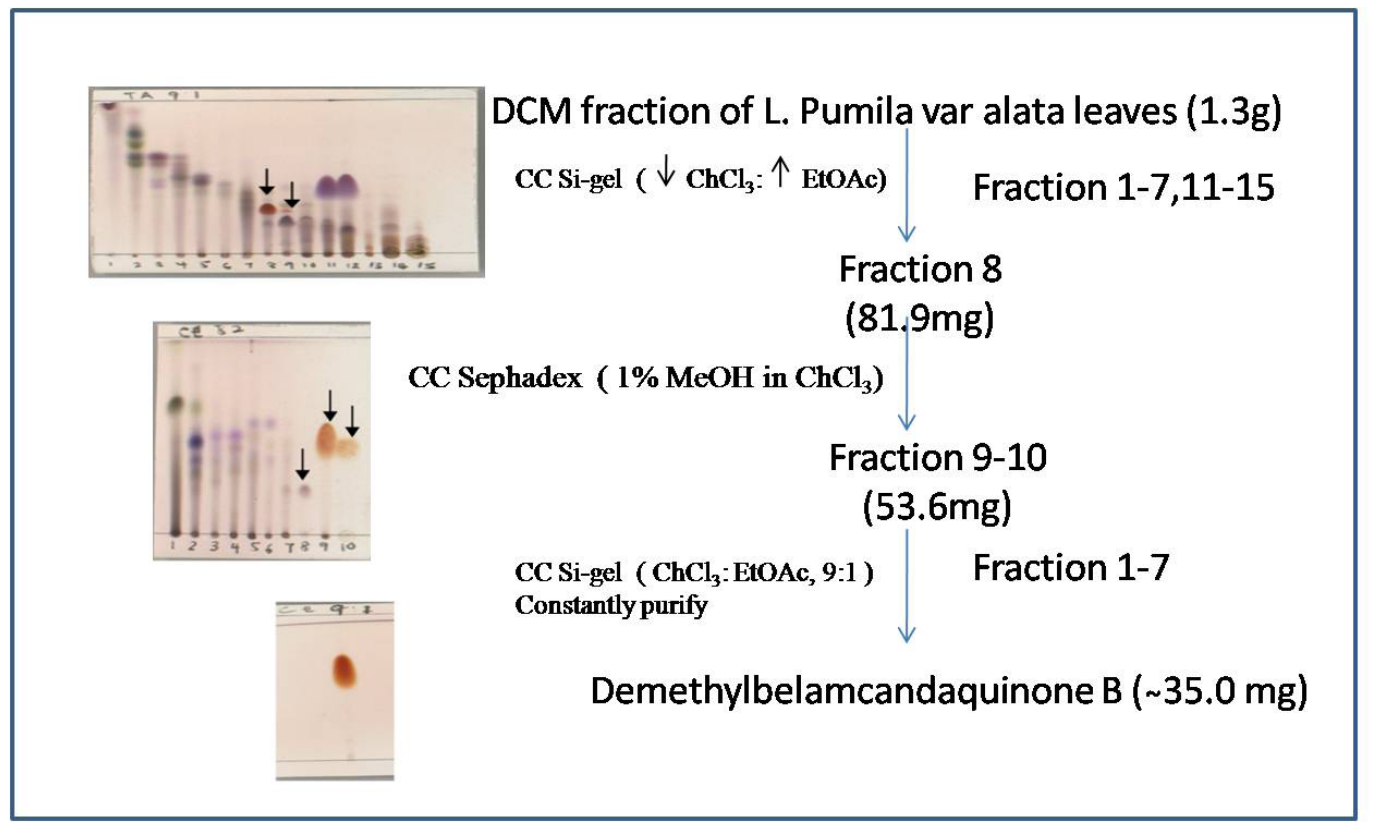

Fig. 1: Isolation of Demethylbelamcandaquinone B from dichloromethane (DCM) fraction of LPva crude aqueous extract.

\section{Alizarin Red S staining}

Alizarin Red S staining was performed to examine extracellular matrix calcium deposits for evaluation of osteoblastic differentiation and mineralization in the culture (Wang et al., 2006). A total of $5 \times 10^{3}$ cells/well were seeded in a 24-well plate. Treatments with differentiation medium were given once every three days. Staining of calcium was then photographed on Day 18. After treatments, cells were briefly rinsed with PBS and fixed with $70 \%$ ethanol. The cells were stained with $40 \mathrm{mM}$ Alizarin red solution ( $\mathrm{pH}$ 4.2) (Sigma, St. Louis, MO, USA) for 30 minutes at room temperature and rinsed with ultrapure water twice. The images of stained cells were captured using an inverted microscope.

\section{Von Kossa staining}

Phosphate ions in the matrix were visualized with von Kossa staining, which is used for determination of mineralization in the culture (Wang et al., 2006). After treatments, cells were washed with PBS and fixed with 10\% formaldehyde for 10 minutes. The cells were rinsed with ultrapure water and incubated with 5\% silver nitrate under ultraviolet light for 1 hour. The cells were then rinsed with ultrapure water. Images of the stained cells were captured using an inverted microscope.

\section{Quantitative calcium deposit assay}

For quantification of insoluble calcium in the matrix layer of the cells (Subbiah et al., 2015), the cultures were decalcified with 0.6 M hydrochloric acid. Calcium in the supernatant was measured with a calcium assay kit (Chemicon International, USA). The absorbance of soluble calcium was measured at 612 $\mathrm{nm}$ with a microtiter plate reader (Tecan, Austria).

\section{Statistical analysis}

Each experiment was carried out in triplicates with at least 3 independent cultures with comparable results. Data were reported as mean $\pm \mathrm{SEM}$ of at least three experiments. All of the data were statistically analyzed using the Statistical Package for Social Sciences (SPSS) software version 20 (IBM, New York, USA). Comparisons between groups were made using ANOVA. $p<0.05$ followed by Tukey's HSD test as a post-hoc test was considered statistically significant.

\section{RESULTS}

\section{Identification of bioactive compound}

Based on the analysis of mass spectrometry and NMR spectra, the compound was established as demethylbelamcandaquinone $\mathrm{B}$ with a molecular formula of $\mathrm{C}_{43} \mathrm{H}_{66} \mathrm{O}_{5}$, as determined from its molecular ion $[\mathrm{M}+\mathrm{H}]^{+}$peak at $m / z$ 663.4883. Demethylbelamcandaquinone B; $35.0 \mathrm{mg}$; orange and waxy $\left(\mathrm{CHCl}_{3}\right)$; UV (EtOH) $\lambda_{\max } \mathrm{nm}(\log \varepsilon): 213$ (3.16), 275 (3.04); IR (ATR) $v_{\max }, \mathrm{cm}^{-1}: 3275,2922,2854,1680,1638,1618,1600$, $1456,1339,1226,1147,1051,847,722$; positive HRESI-MS m/z: $663.4883[\mathrm{M}+\mathrm{H}]^{+}\left(\mathrm{C}_{43} \mathrm{H}_{66} \mathrm{O}_{5}, \mathrm{MW} 662.9811\right)$ (Figure 2); ${ }^{1} \mathrm{H}-\mathrm{NMR}$ $\left(600 \mathrm{MHz} ; \mathrm{CDCl}_{3}\right) \delta_{\mathrm{H}}(\mathrm{ppm}): 0.89$ (6H, m, H-21, H-21'), $1.33-$ 1.18 (18H, m, H-8-H-14, H-19-H-20, H-8'-H-14', H-19'-H-20', overlapped ), 2.01 (8H, $m, \mathrm{H}-15, \mathrm{H}-18, \mathrm{H}-15$ ', H-18'), 2.18 (H, $m$, H-7) 2.35 ( $m, \mathrm{H}-7), 2.23$ (2H, $m, \mathrm{H}-7$ '), 3.85 ( $s, \mathrm{OMe}), 5.34(4 \mathrm{H}$, $m, \mathrm{H}-16-\mathrm{H}-17, \mathrm{H}-16$ '-H-17'), 5.99 ( $b r s, \mathrm{OH}), 6.16$ (d, $J=1.8 \mathrm{~Hz}$, H-6'), 6.29 ( $d, J=1.8 \mathrm{~Hz}, \mathrm{H}-4$ '); ${ }^{13} \mathrm{C}-\mathrm{NMR}\left(150 \mathrm{MHz} ; \mathrm{CDCl}_{3}\right)$ $\delta_{\mathrm{C}}(\mathrm{ppm}): 14.0\left(\mathrm{C}-21, \mathrm{C}-21^{\prime}\right), 22.4$ (C-20, C-20'), 26.9 (C-18, C-18'), 27.2 (C-15, C-15'), 28.2 (C-7), 33.5 (C-7'), 29.0-30.0 (C-8-C-14), (31.8 (C-19), 32.0 (C-19'), 56.3 (OMe), 129.9 (C-16- 
C-17, C-16'-C-17'), quinone ring; 182.2 (C-1), 146.9 (C-2), 141.0 (C-3), 188.0 (C-4), 107.4 (C-5), 158.9 (C-6), benzene ring; 153.7 (C-1'), 112.2 (C-2'), 143.2 (C-3'), 108.2 (C-4'), 156.7 (C-5'), 100.9 (C-6') (Figure 3).

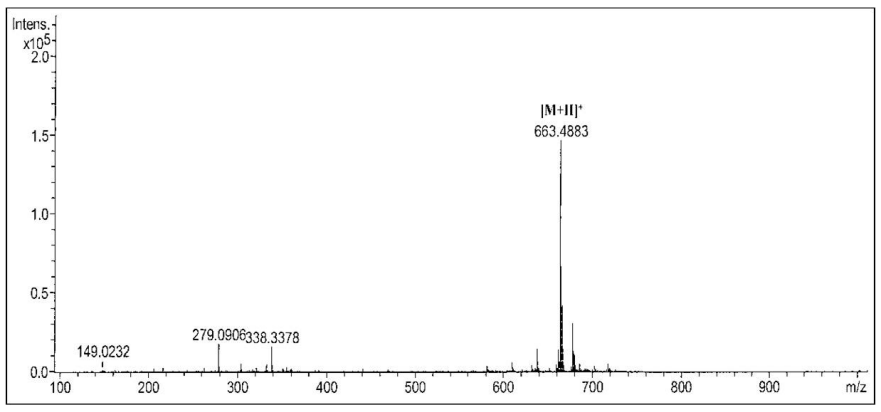

Fig. 2: Mass Spectrum of Demethylbelamacandaquinone B.

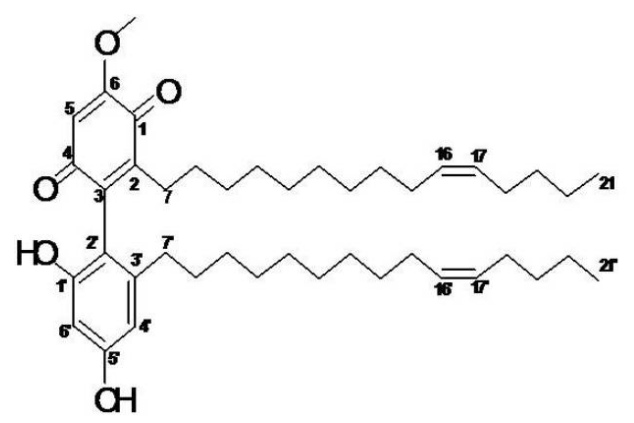

Fig. 3: Chemical structure of Demethylbelamcandaquinone B.

\section{Cell viability}

During treatments, cells were cultured with differentiation media to initiate cell differentiation. The percentage of viable cells was significantly higher $(p<0.05)$ when treated with the crude aqueous extract of LPva at concentrations of $10-100 \mu \mathrm{g} / \mathrm{ml}$ after 24 hours of treatment. However, there was no significant increase in cell viability after 72 hours of treatment with LPva crude aqueous extract (Figure 4). Among the three fractions, DCM fraction had significantly higher percentage of cell viability at concentrations of 5 to $50 \mu \mathrm{g} / \mathrm{ml}$ after 24 and 72 hours of treatment when compared to other fractions (Table 1). Interestingly, demethylbelamcandaquinone $\mathrm{B}$ isolated from DCM fraction of LPva crude aqueous extract exhibited a higher percentage of viable cells at low concentrations $(0.02-0.6 \mu \mathrm{g} /$ $\mathrm{ml}$ ) compared to aqueous extract and DCM fraction of LPva. For the positive control (treated with 17- $\beta$ estradiol), the percentage of viable cells was significantly increased at concentrations of 0.002 to $0.08 \mu \mathrm{g} / \mathrm{ml}$. The continued proliferation of MC3T3-E1 osteoblast cells after 72 hours of treatment with DCM fraction and demethylbelamcandaquinone B (active compound) indicated that the MC3T3-E1 number remained to be increased even during the differentiation phase.

\section{Mineralization}

The differentiating and mineralizing activities of the aqueous extract, DCM fraction and demethylbelamcandaquinone $\mathrm{B}$ (active compound) of LPva were assessed by staining with Alizarin Red S (Figure 5) and Von Kossa (Figure 6) at day-
18. It was found that all treatments were able to stimulate the differentiation and mineralization of MC3T3-E1 cell culture in a dose-dependent manner. Similar to that seen in bone, cartilage, and teeth, calcium-phosphate, a substitute of hydroxyapatite, was deposited in the mineral phase of the culture. The calcium levels of the LPva treated-groups were significantly higher compared to the normal control group and at par with $17 \beta$ estradiol (positive control) (Figure 7).

\section{DISCUSSION}

Demethylbelamcandaquinone B was obtained as an orange wax. The HRESI-MS spectrum of interest compound showed a protonated molecular ion $[\mathrm{M}+\mathrm{H}]^{+}$peak at $\mathrm{m} / z$ 663.4883, suggesting its molecular formula as $\mathrm{C}_{43} \mathrm{H}_{66} \mathrm{O}_{5}$. The ATR-FTIR spectrum displayed absorptions at $3275 \mathrm{~cm}^{-1}$, indicating the presence of hydroxyl groups. The absorption at $1680-1639 \mathrm{~cm}^{-1}$ showed presence of carbonyl and double bond groups in the skeleton of demethylbelamqandaquinone B. The ${ }^{1} \mathrm{H}-$ and ${ }^{13} \mathrm{C}$ $\mathrm{NMR}\left(600 \mathrm{MHz}, \mathrm{CDCl}_{3}\right)$ spectra exhibited presence of a methoxy$p$-benzoquinone ring $\left[\delta_{\mathrm{C}} 182.2(\mathrm{C}-1), 146.9(\mathrm{C}-2), 140.9(\mathrm{C}-3)\right.$, 187.9 (C-4), 107.4 (C-5), 158.9 (C-6), 56.3 (O-Me) ppm and $\delta_{\mathrm{H}}$ $5.99(\mathrm{~s}, \mathrm{H}-5), 3.85(\mathrm{~s}, \mathrm{O}-\mathrm{Me}) \mathrm{ppm}]$ and a tetra-substituted benzene ring $\left[\delta_{\mathrm{C}} 153.7(\mathrm{C}-1\right.$ '), 112.2 (C-2'), 143.2 (C-3'), 108.2 (C-4'), 156.7 (C-5'), 100.9 (C-6') ppm and $\delta_{\mathrm{H}} 6.29$ (d, H-4', $J=1.8 \mathrm{~Hz}$ ), $6.16(\mathrm{~d}, \mathrm{H}-6$ ' $J=1.8 \mathrm{~Hz}$ ) ppm], respectively. The two rings had a linkage between C-3 and C-2', and each ring had a long alkenyl chain (15 carbons). The substitution pattern and linkage between the two rings were confirmed by HMBC correlation as well as comparison with literature (Zulfiqar and Ikhlas, 2011; Nabil et al., 2012). The chemical shifts of methylene carbons adjacent to the double bonds at $\delta_{\mathrm{C}} 26-28$ (C-15, C-15', C-18, C-18') in the ${ }^{13} \mathrm{C}$ NMR spectrum supported the $Z$-geometry of the double bonds in the side chain (Gao et al., 2008). The chemical shifts assignment was confirmed by the HMBC correlations.

Bone remodeling involves a balance of bone formation and resorption, which is an essential physiological process in the maintenance of bone mass. Osteoporosis occurs when bone resorption exceeds bone formation. An ideal way to prevent agerelated bone loss is to promote bone formation (Drake et al., 2015). Therefore, studies are on-going to find new agents that have the potential for promoting osteoblastogenesis to counteract bone loss related to aging.

For osteoporosis studies, LP was shown to increase antioxidant enzymes activities, superoxide dismutase and gluthathion peroxidise levels and lowered oxidative stress marker, malondialdehye level in estrogent-deficient rat models (Effendy and Shuid, 2014). In bone remodeling, LP was demonstrated to stimulate OPG production and reduced RANKL gene expression (Fathilah et al., 2013). Shuid et al. (2011) demonstrated that LPva was able to increase the secretion of osteocalcin, a phenotypic marker for the late stage of osteoblast differentiation. Several in vivo studies showed that aqueous LPva extracts improved femoral strength (Fathilah et al., 2012a) and increased bone formation rate, in terms of increased osteoblast number, osteoid surface and volume, and decreased osteoclast surface (Fathilah et al., 2012b). Micro-CT analyses revealed that treatment with aqueous LPva extract and exogenous estrogen reversed osteoporotic changes in estrogen-deficient rats in terms of trabecular bone volume, 
thickness, separation, and number (Effendy et al., 2014). These results showed that LPva bone protective action was at par with HRT. Based on the studies, it can be deduced that LPva prevented bone loss by promoting bone formation and suppressing bone resorption.
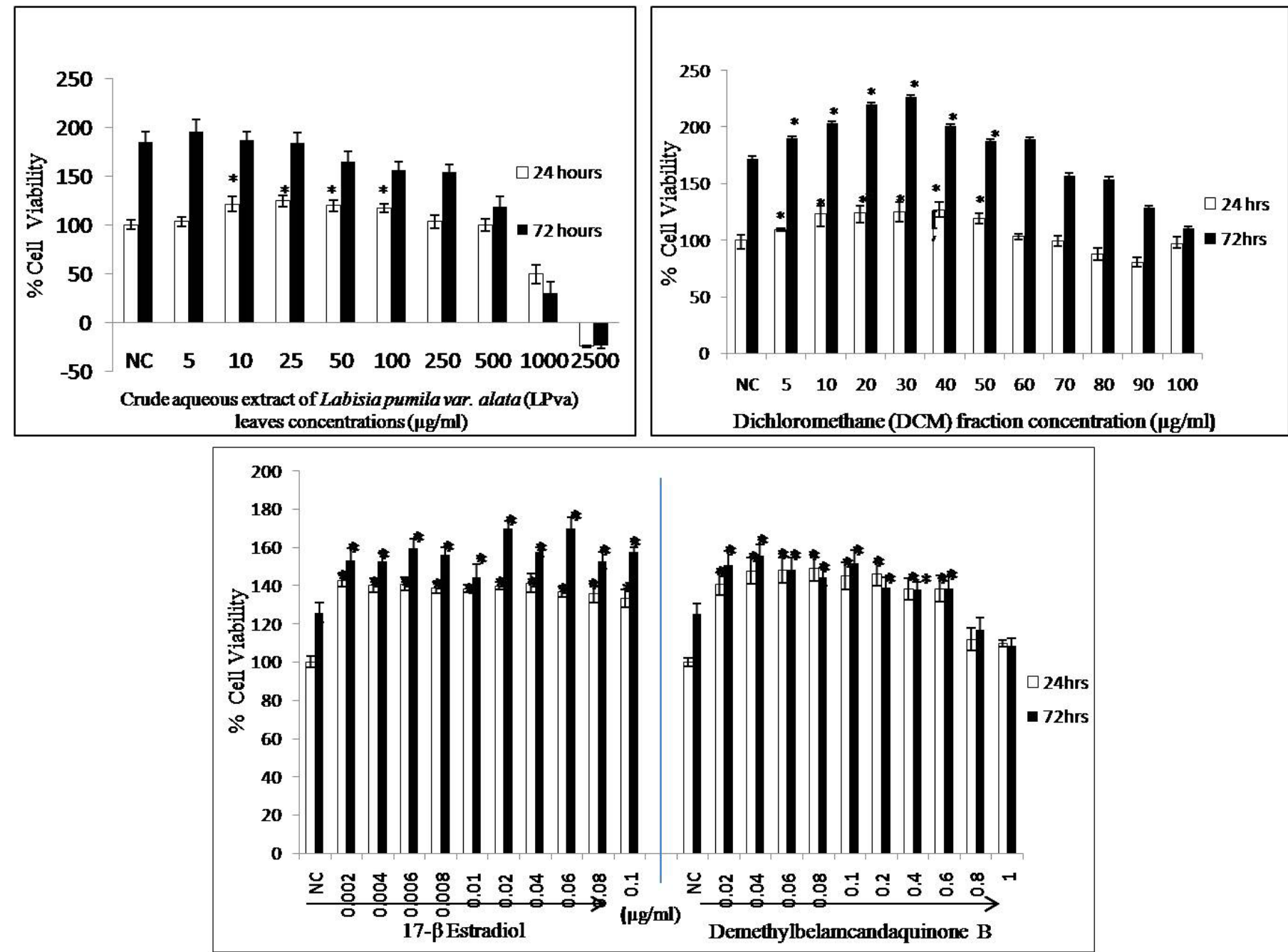

Fig. 4: Effects of LPva crude aqueous extracts, dichloromethane (DCM) fraction, 17- $\beta$ estradiol and demethylbelamcandaquinone B on viable MC3T3-E1 pre-osteoblast cells. * significantly increase compared to normal control (NC).

Table 1: Effects of Hexane (Hex), dichloromethane (DCM) and methanol (Met) fraction on viable MC3T3-E1 osteoblast cells. DCM fraction was able to increase viable cells at the concentration of 5-50 $\mu \mathrm{g} / \mathrm{ml}$ after 24 and 72 hours treatment. Comparing Hex and Met fractions, these fractions were increased the viable cells at the concentration of $5-50 \mu \mathrm{g} / \mathrm{ml}$ only after 72 hours of treatment. * significantly increase compared to normal control (NC).

\begin{tabular}{|c|c|c|c|c|c|c|}
\hline & Hex (24 hrs) & Hex (72 hrs) & DCM (24 hrs) & DCM (72 hrs) & Met (24 hrs) & Met (72 hrs) \\
\hline NC & $100 \pm 1.583$ & $165.08 \pm 15.508$ & $100 \pm 4.506$ & $172.25 \pm 7.785$ & $100 \pm 3.11$ & $123.87 \pm 3.242$ \\
\hline 5 & $104.63 \pm 5.605$ & $180.63 \pm 5.89^{*}$ & $109.22 \pm 1.433$ & $190.31 \pm 1.73$ & $109.34 \pm 0.99$ & $178.26 \pm 3.657^{*}$ \\
\hline 10 & $98.37 \pm 4.848$ & $213.76 \pm 4.835^{*}$ & $123.34 \pm 6.937^{*}$ & $203.23 \pm 10.98^{*}$ & $91.08 \pm 3.158$ & $156.55 \pm 5.324^{*}$ \\
\hline 20 & $108.81 \pm 4.411$ & $231.24 \pm 20.63^{*}$ & $124.39 \pm 5.863^{*}$ & $219.81 \pm 8.94 *$ & $103.44 \pm 3.91$ & $159.62 \pm 4.66^{*}$ \\
\hline 30 & $100.44 \pm 4.388$ & $216.73 \pm 11.738^{*}$ & $124.75 \pm 8.496^{*}$ & $226.61 \pm 8.514 *$ & $91.13 \pm 4.432$ & $158.39 \pm 2.966^{*}$ \\
\hline 40 & $105.25 \pm 5.957$ & $205.21 \pm 7.32 *$ & $126.75 \pm 7.208^{*}$ & $200.71 \pm 6.804^{*}$ & $93.58 \pm 3.78$ & $150.23 \pm 7.1^{*}$ \\
\hline $\mathbf{5 0}$ & $84.53 \pm 2.888$ & $196.05 \pm 14.003^{*}$ & $119.33 \pm 4.174 *$ & $187.40 \pm 5.079^{*}$ & $103.87 \pm 7.624$ & $144.75 \pm 6.823^{*}$ \\
\hline 60 & $99.22 \pm 4.69$ & $188.66 \pm 15.073$ & $103.45 \pm 2.261$ & $189.12 \pm 3.079^{*}$ & $88.53 \pm 6.726$ & $126.81 \pm 3.849$ \\
\hline 70 & $98.58 \pm 2.979$ & $174.67 \pm 13.71$ & $99.15 \pm 4.667$ & $157.23 \pm 4.829$ & $98.14 \pm 4.711$ & $131.74 \pm 1.993$ \\
\hline 80 & $98.24 \pm 5.681$ & $162.75 \pm 10.409$ & $88.01 \pm 4.69$ & $154.07 \pm 5.505$ & $102.21 \pm 4.556$ & $119.57 \pm 3.22$ \\
\hline 90 & $93.23 \pm 2.337$ & $153.49 \pm 5.865$ & $80.26 \pm 4.747$ & $128.48 \pm 3.754$ & $100.49 \pm 6.096$ & $114.23 \pm 4.092$ \\
\hline 100 & $81.99 \pm 7.171$ & $136.79 \pm 3.058$ & $97.17 \pm 5.878$ & $110.08 \pm 4.119$ & $80.29 \pm 3.139$ & $87.75 \pm 2.26$ \\
\hline
\end{tabular}




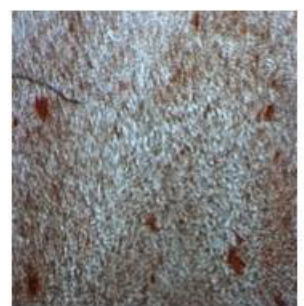

Normal Control(NC)

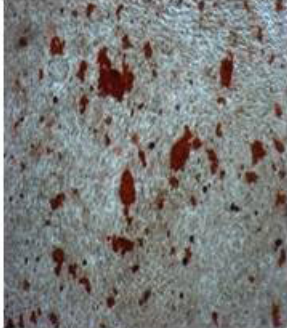

Crude aqueous extract $10 \mu \mathrm{g} / \mathrm{ml}$

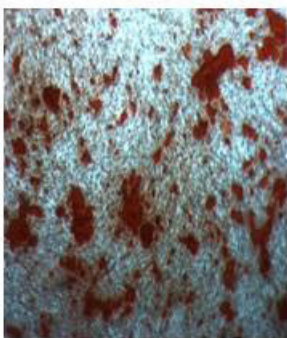

Demethylbelamca ndaquinone $B 0.4$ $\mu \mathrm{g} / \mathrm{ml}$
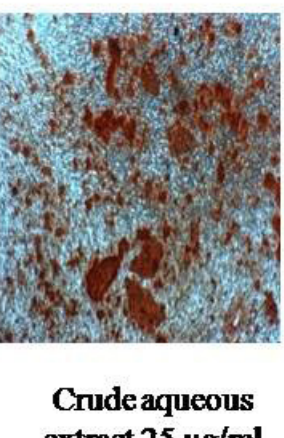
extract $25 \mu \mathrm{g} / \mathrm{ml}$

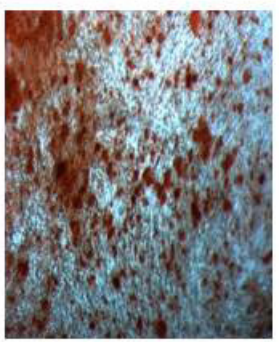

Demethylbelamca
ndaquinoneB 0.6
$\mu \mathrm{g} / \mathrm{ml}$

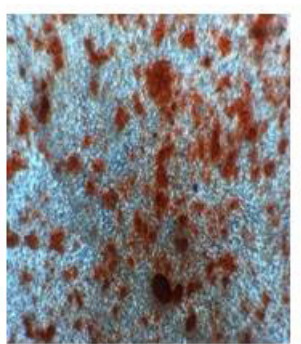

Dem $5 \mu \mathrm{g} / \mathrm{ml}$

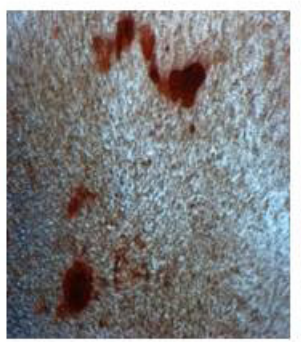

17B estradiol 0.04 $\mu \mathrm{g} / \mathrm{ml}$

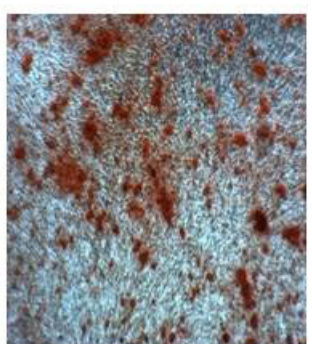

Dcm $10 \mu \mathrm{g} / \mathrm{ml}$

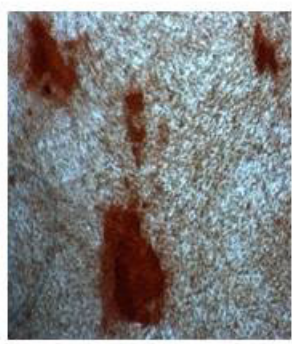

17B estradiol 0.06

$\mu \mathrm{g} / \mathrm{ml}$

\section{Magnification: 100x}

Fig. 5: Effects of LPva treatments on mineralized nodule formation in MC3T3-E1 cells. Calcium deposits appear bright red in Alizarin Red S Staining (magnification 100x). Cells treated with LPva treatments for 18 days.

The present study investigated the effects of crude aqueous extract, fractions and an isolated active compound of LPva leaves on the viability of mouse pre-osteoblast (MC3T3-E1) cells in vitro. An initial study indicated that crude aqueous extract of LPva exhibited proliferative properties on MC3T3-E1 cells. This extract was fractionated and the active fraction identified by observing its proliferative properties on MC3T3-E1 cells before finally identifying its active compound. The findings demonstrated that crude aqueous extract, DCM fraction and its compound were non-toxic to MC3T3-E1 pre-osteoblast cells even after 72 hours of treatment. In the first round, the crude aqueous extract was fractionated into three solvents according to polarity. DCM fraction significantly enhanced MC3T3-E1 cell proliferation compared to normal control and other solvent fractions. Therefore, the DCM fraction was considered to be the active fraction which contained the bioactive compound responsible for enhancing the proliferative rate of MC3T3-E1 cells. Moreover, a previous study reported that DCM fraction of LP was able to selectively inhibit CYP2C activities, which may be attributed to the presence of lipophilic yet slightly polar compounds within the LP extracts (Pan et al., 2012). The TLC analysis showed the high presence of orange spots in DCM fraction, which was suspected to be the bioactive compound. Thus, the DCM fraction was subfractionated on a silica gel column and Sephadex LH20, which yielded an active compound. This compound tested positive for the proliferation of MC3T3-E1 cells. By using LCMS and NMR spectroscopy, demethylbelamcandaquinone B was elucidated to be the active compound. It contains structural determinants such as hydrophilic (dihydroxybenzene ring) and hydrophobic (aliphatic chain) regions which have strong amphiphilic character (Kozubek and Tyman, 1999). Its chemical structure enabled it to incorporate with phospholipid membrane from the interaction of free hydroxyl groups in the resorcinol ring with phospholipids through the formation of hydrogen bonds within the membrane (Kozubek and Tyman, 1999). Its core chemical structure is similar to that of genistein, quercetin, and chrysin, which are popularly known as a phytoestrogen. Genestein and quercetin were known to have stronger estrogen binding affinity to estrogen receptor beta 
(ERß) (Han et al., 2002). Thus, demethylbelamqandaquinone B has the potential to exert endogenous estrogen action. Although it has a long aliphatic chain which may influence its binding to the estrogen receptor, it was able to exhibit potent proliferation effects on MC3T3-E1 cells. Besides, demethylbelamcandaquinone B was found to act at low concentrations than the crude aqueous extract and DCM fraction, which was at 0.02 to $0.8 \mu \mathrm{g} / \mathrm{ml}$. Similar to the crude extract and DCM fraction of LPva, demethylbelamcandaquinone $B$ also affected osteoblast differentiation with increased calcium and phosphate contents in bone mineralization. As for other properties, demethylbelamcandaquinone $\mathrm{B}$ was shown to have cardioprotective effects towards isoproterenol-induced myocardial infarction in rats (Dianita et al., 2015). The isolation of the active compound from the leaves of LPva plant, instead of the root or stem, meant a sustainable and economical production of raw material could be obtained repeatedly without having to kill the plant. The advanced isolation of active compound from crude extract using a combination of chromatography and spectroscopy techniques led to an interest in the plant as a natural source of the anti-osteoporotic compound, alternative to hormonal replacement therapy. Demethylbelamcandaquinone B may act as a selective estrogen receptor modulator (SERM). Gene expression studies are on the way to determine its mechanism of action.

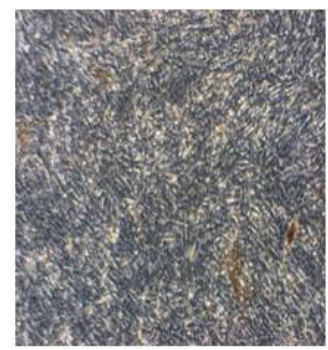

Normal Control(NC)

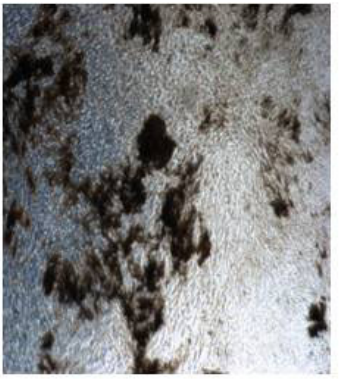

Crude aqueous extract 10 $\mu \mathrm{g} / \mathrm{ml}$

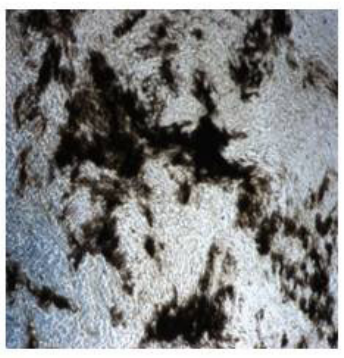

Demethylbelamcandaqu inone $B \quad 0.4 \mu \mathrm{g} / \mathrm{ml}$

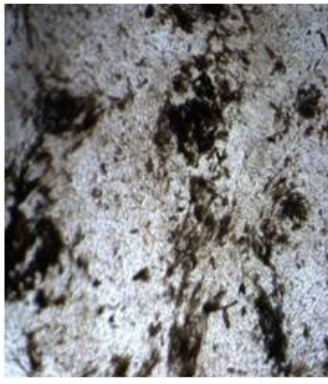

Crude aqueous extract $25 \mu \mathrm{g} / \mathrm{ml}$

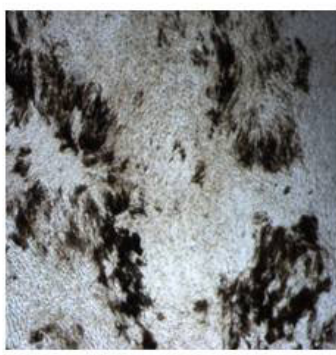
Demethylbelamcandaqu inone $B \quad 0.6 \mu \mathrm{g} / \mathrm{ml}$

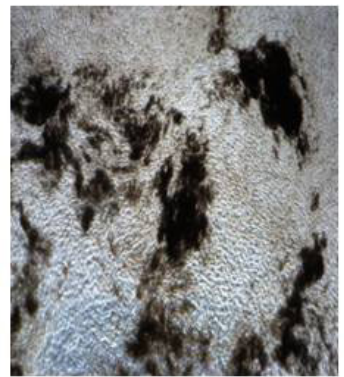

Dcm $5 \mu \mathrm{g} / \mathrm{ml}$

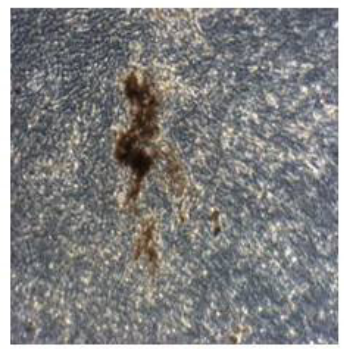

17Bestradiol 0.04
$\mu \mathrm{g} / \mathrm{ml}$

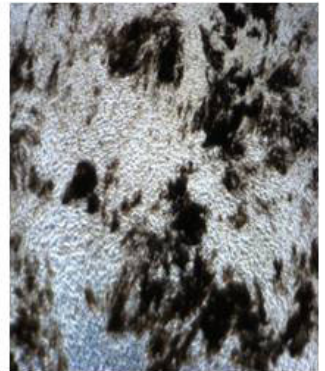

Dem $10 \mu \mathrm{g} / \mathrm{ml}$

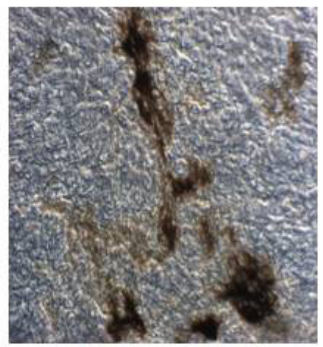
17B estradiol 0.06
$\mu \mathrm{g} / \mathrm{ml}$

\section{Magnification: $100 x$}

Fig. 6: Effects of LPva treatments on mineralized nodule formation in MC3T3-E1 cells. Phosphate precipitation appears black in Von Kossa Staining (magnification 100x). Cells treated with LPva treatments for 18 days.

\section{CONCLUSION}

Demethylbelamcandaquinone $B$ isolated from the DCM fraction of LPva leaves is a possible candidate for further development as an anti-osteoporotic agent.

\section{ACKNOWLEDGMENT}

This study was supported by NKEA Research Grant Scheme (NRGS) (NH1113D018-2). The authors would also like to acknowledge the Faculty of Medicine and Faculty of Pharmacy, 
Universiti Kebangsaan Malaysia (UKM) for providing resources and tremendous support. We are grateful to Ms. Nurul Hafiza Abas from the Department of Pharmacology, Faculty of Medicine UKM, for her technical assistance.

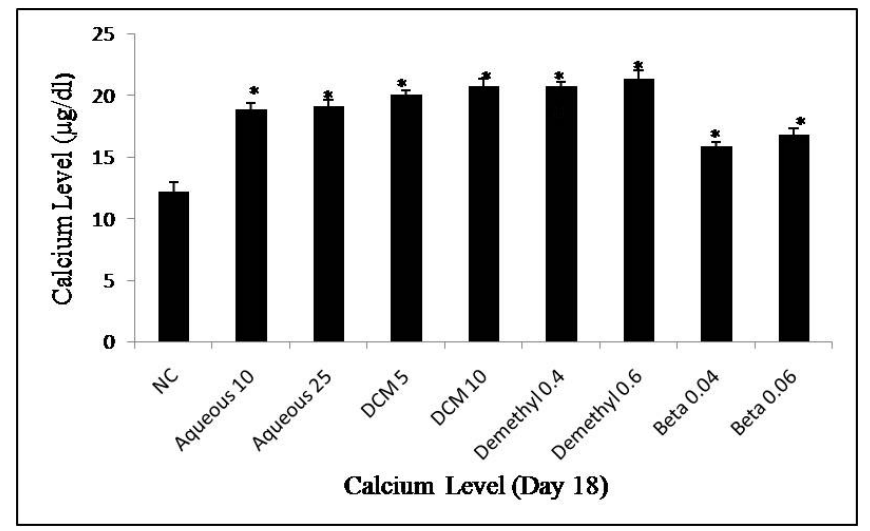

Fig. 7: Effects of LPva treatments on calcium level. Cells treated with LPva treatments for 18 days. * significantly increase compared to normal control (NC).

\section{CONFLICT OF INTEREST}

The authors declare that they have no conflict of interests.

\section{REFERENCES}

Atteritano M, Mazzafero S, Frisina A, Cannata ML, Bitto A, D'Anna R, Squadrito F, Frisina N. Genistein effects on quantitative ultrasound parameters and bone mineral density in postmenopausal women. Osteoporos Int, 2009; 11:1947-1954.

Avula B, Wang YH, Ali Z, Smillie TJ, Khan IA. Quantitative determination of triperpene saponins and alkenated-phenolics from Labisia pumila using an LCUV/ELSD method and confirmation by LC-ESI-TOF. Planta Med, 2011; 77:1742-1748.

Bassuk SS, Manson JE. The timing hypothesis: Do coronary risks of menopausal hormone therapy vary by age or time since menopause onset? Metabolism, 2016; 5:794-803.

Bord S, Beavan SR. The effects of estrogen on osteoprotegerin, RANKL, and estrogen receptor expression in human osteoblasts. Bone, 2003; 32:136-141.

Chlebowski RT, Anderson GL, Sarto GE, Haque R, Runowicz $\mathrm{CD}$, Aragaki AK, Thomson CA, Howard BV, Wactawski-Wende J, Chen C, Rohan TE, Simon MS, Reed SD, Manson JE. Continuous Combined Estrogen Plus Progestin and Endometrial Cancer: The Women's Health Initiative Randomized Trial. J Natl Cancer Inst, 2016; 3:350-359.

Deyhima F, Stoeckerb BJ, Brusewitzc GH, Arjmandib BH. The effects of estrogen depletion and isoflavones on bone metabolism in rats Nutr Res, 2003; 23:123-130.

Dianita R, Jantan I, Amran AZ, Jalil J. Protective effects of Labisia pumila var alata on biochemical and histopathological alterations of cardiac muscle cells in isoproterenol-induced myocardial infarction rats. Molecules, 2015; 3:4746-4763.

Drake T, Clarke B, Lewiecki EM. The pathophysiology and treatment of osteoporosis. Clin Ther, 2015; 8:1837-1850.

Effendy NM, Shuid AN. Time and dose-dependent effects of Labisia pumila on bone oxidative status of postmenopausal osteoporosis rat model. Nutrients, 2014; 8:3288-3302.

Effendy NM, Khamis MF, Soelaiman IN, Shuid AN. The effects of Labisia pumila on postmenopausal osteoporotic rat model: Dose and time-dependent micro-CT analysis. Journal of X-ray Science and Technology, 2014; 24:503-518.

Fathilah SN, Mohamed N, Muhammad N, Mohamed IN, Soelaiman IN, Shuid AN. Labisia pumila regulates bone-related genes expressions in postmenopausal osteoporosis model. BMC Complement Altern Med, 2013; 1:217-523.

Fathilah SN, Abdullah S, Mohamed N, Shuid AN. Labisia pumila prevents complications of osteoporosis by increasing bone strength in a rat model of postmenopausal osteoporosis. Evid Based Complement Alternat Med, 2012a; ID: 948080.

Fathilah SN, Shuid AN, Mohamed N, Muhammad N, Soelaiman IN. Labisia pumila protects the bone of estrogen-deficient rat model: A histomorphometric study. J Ethnopharmacol, 2012b; 1:294-299.

Fazliana M, Wan Nazaimoon WM, Gu HF, Östenson CG. Labisia pumila extract regulates body weight and adipokines in ovariectomized rats. Maturitas, 2009; 1:91-97.

Feng X, Mcdonald JM. Disorder of bone remodelling. Annu. Rev Pathol, 2011; 6:121-145.

Gao Z, Ali Z, Khan IA. Glycerogalactolipids from the fruit of Lyciumbarbarum. Phytochemistry, 2008; 69:2856-2861.

Guo AJ, Choi RC, Zheng KY, Chen VP, Dong TT, Wang Z, Vollmer G, Lau DT, Tsim KW. Kaempferol as a flavonoid induces osteoblastic differentiation via estrogen receptor signalling. Chin Med, $2012 ; 7: 10-16$

Han DH, Denison MS, Tachibana H, Yamada K. Relationship between estrogen receptor-binding and estrogenic activities of environmental estrogens and suppression by flavonoids. Biosci Biotechnol Biochem, 2002; 7:1479-1487.

Kanis JA, Johnell O. An estimate of the worldwide prevalence and disability associated with osteoporotic fractures. Osteoporos Int, 2006; 12:1726-1733.

Karimi E, Jaafar HZ, Ahmad A. Antifungal, anti-inflammatory and cytotoxicity activities of three varieties of Labisia Pumila Benth: from microwave obtained extracts. BMC Complement Altern Med, 2013; 1:2029.

Karimi E, Jaafar HZ, Ahmad S. Phytochemical analysis and antimicrobial activities of methanolic extracts of leaf, stem and root from different varieties of Labisa pumila Benth. Molecules, 2011; 6:4438-4450.

Kotsopoulos J, Huzarski T, Gronwald J, Moller P, Lynch HT, Neuhausen SL, Senter L, Demsky R, Foulkes WD, Eng C, Karlan B, Tung $\mathrm{N}$, Singer CF, Sun P, Lubinski J, Narod SA. Hormone replacement therapy after menopause and risk of breast cancer in BRCA1 mutation carriers: a case-control study. Breast Cancer Res Treat, 2016; 2:365-373.

Kozubek A, Tyman HP. Resorcinolic lipids, the natural nonisoprenoid phenolic amphiphiles and their biological activity. Chemical Review, 1999; 99:1-24.

Lina Wati D, Khor SC, Tan JC, Chua KH, Yasmin Anum MY, Suzana M. Piper betle L. modulates senescence-associated genes expression in replicative senescent human diploid fibroblasts. Biomed Res Int, 2017; ID: 6894026.

Maximov PY, Lee TM, Jordan VC. The discovery and development of selective estrogen receptor modulators (SERMs) for clinical practice. Curr Clin Pharmacol, 2013; 2:135-155.

McCann RM, Colleary G, Geddis C, Clarke SA, Jordan GR, Dickson GR, Marsh D. Effect of osteoporosis on bone mineral density and fracture repair in a rat femoral fracture model. J Orthop Res, 2008; 26:384393

Mohamad N, Mahmood M, Mansor H. Antioxidative properties of leaf extracts of a popular Malaysian herb, Labisia pumila. J Med Plants Res, 2009; 3(4):217-223.

Muhammad Z, Mustafa AM. 1994. Traditional Malay medicinal plants. Kuala Lumpur: Fajar Bakti Sdn Bhd. 2.

Nabil AA, Khozirah S, Faridah A, Ralf K, Ethel JJ, Johnson S, Naoshi Y, Toshio H, Nordin HL. Alkenylresorcinols and cytotoxic activity of the constituents isolated from Labisia pumila. Phytochemistry, 2012; 80:42-49.

Nor Ashila A, Jamia Azdina J, Noraini T, Nur Ain MH, Mohd Ruzi AR, Carla WS, Kartiniwati M, Khairana H, Juriyati J. Comparative study of three Marantodes pumilum varieties by microscopy, spectroscopy and chromatography. Brazilian Journal of Pharmacognosy, 2016; 26(1):1- 
14.

Pan Y, Tiong KH, Abd-Rashid BA, Ismail Z, Ismail R, Mak JW, Ong EO. Inhibitory effects of cytochrome P450 enzymes CYP2C8, CYP2C9, CYP2C19 and CYP3A4 by Labisia pumila extracts. J Ethnopharmacol, 2012; 143:586-591.

Pisani P, Renna MD, Conversano F, Casciaro E, Di Paola M, Quarta E, Muratore M, Casciaro S. Major osteoporotic fragility fractures: Risk factor updates and societal impact. World J Orthop, 2016; 3:171-181.

Samuagam L, Akowuah GA, Okechukwu PN. Partial purification and antinociceptive investigation of extracts of leaves of Labisia pumila. Asian J Pharm Clin Res, 2011; 4(4):44-46.

Sanusi RAM, Ab Shukor NA, Sulaiman MR. Anti-inflammatory Effects of Labisia pumila (Blume) F. Vill-Naves. Aqueous Extract. Sains Malaysiana, 2013; 42(10):1511-1516.

Shuid AN, Ping LL, Muhammad N, Mohamed N, Soelaiman IN. The effects of Labisia pumila var. alata on bone markers and bone calcium in a rat model of post-menopausal osteoporosis. J Ethnopharmacol, 2011; 133(2):538-542.

Singh N, Kumar D, Dubey P, Chandanan A, Singh P. The effects of isoflavones on bone density in postmenopausal women at tertiary centre. Int J Reprod Contracept Obstet Gynecol, 2015; 6:1763-1765.

Sorensen JMG, Henriksen K, Dziegiel MH, Tankó LB, Karsdal MA. Estrogen directly attenuates human osteoclastogenesis, but has no effect on resorption by mature osteoclasts. DNA Cell Biol, 2006; 25:475483.

Stone BC. New and noteworthy Malesian Myrsinaceae III. On the genus Ardisia Sw. Borneo. Proc. Acad. Natl. Sci. Phila, 1989; 141: 263-306.

Subbiah R, Suhaeri M, Hwang P, Kim W, Kwideok P. Investigation of the changes of biophysical/ mechanical characteristics of differentiating preosteoblasts in vitro. Biomaterial Research, 2015; 19:24.

Ueda M, Horiguchi Y, Sugimoto M, Ikeda S, Kume S. Effects of coumestrol administration to maternal mice during pregnancy and lactation on renal Ca metabolism in neonatal mice. Animal Science Journal, 2011; 6:469-473.

Wahab N, Yusoff W, Shuib A, Wan N, Khatiza H. Labisia pumila has similar effects to estrogen on the reproductive hormones of ovarectomized rats. Internet Journal of Herbal and Plant Medicine, 2011; 2:1-6.

Wang Y, Liu Y, Maye P, Rowe DW. Examination of mineralized nodule formation in living osteoblastic cultures using fluorescent dyes. Biotechnol Prog, 2006; 6:1697-1701.

Xu X, Gou W, Wang A, Wang Y, Guo Q, Lu Q, Lu S, Peng J. Basic research and clinical applications of bisphosphonates in bone disease: what have we learned over the last 40 years? J Transl Med, 2013; 11:303310 .

Yan Z, Yang W, Yang F, Kersten-Niessen M, Jansen JA, Both SK. Effects of continuous passaging on mineralization of MC3T3-E1 cells with improved osteogenic culture protocol. Tissue Eng Part C Methods, 2014; 3:198-204.

Zhu Y, Kawaguchi K, Kiyama R. Differential and directional estrogenic signaling pathways induced by enterolignans and their precursors. PLoS One, 2017; 2:e0171390.

Zulfiqar A, Ikhlas AK. Alkyl phenol and saponins from the roots of Labisia pumila (Kacip Fatimah). Phytochemistry, 2011; 72:2075-2080.

How to cite this article:

Hairi HA, Jamal JA, Aladdin NA, Husin K, Sofi NSM, Mohamed N, Mohamed IN, Shuid AN. Demethylbelamcandaquinone B Isolated from Labisia Pumila Enhanced Proliferation and Differentiation of Osteoblast Cells. J App Pharm Sci, 2018; 8(08): 012-020. 